\title{
Anatomically-Driven Soft-Tissue Simulation Strategy for Cranio-Maxillofacial Surgery Using Facial Muscle Template Model
}

\author{
Hyungmin Kim ${ }^{1}$, Philipp Jürgens ${ }^{2}$, Lutz-Peter Nolte ${ }^{1}$, and Mauricio Reyes ${ }^{1}$ \\ 1 Institute for Surgical Technology and Biomechanics, University of Bern, \\ Stauffacherstrasse 78, 3014 Bern, Switzerland \\ hyungmin.kim@istb.unibe.ch \\ 2 Department of Cranio-Maxillofacial Surgery, University Hospital Basel, \\ Spitalstrasse 21, 4031 Basel, Switzerland
}

\begin{abstract}
We propose a computationally efficient and bio-mechanically relevant soft-tissue simulation method for cranio-maxillofacial (CMF) surgery. A template-based facial muscle reconstruction was introduced to minimize the efforts on preparing a patient-specific model. A transversely isotropic mass-tensor model (MTM) was adopted to realize the effect of directional property of facial muscles in reasonable computation time. Additionally, sliding contact around teeth and mucosa was considered for more realistic simulation. Retrospective validation study with postoperative scan of a real patient showed that there were considerable improvements in simulation accuracy by incorporating template-based facial muscle anatomy and sliding contact.
\end{abstract}

Keywords: soft-tissue simulation, mass-tensor model, craniomaxillofacial surgery.

\section{Introduction}

There has been a comprehensive amount of studies on facial soft-tissue modeling, which can be classified into three categories depending on the computational method: mass-spring model (MSM), finite-element model (FEM), and masstensor model (MTM). MSM was widely accepted for real-time application due to its computational efficiency and geometric simplicity [1] [2] [3, whereas FEM has strong bio-mechanical relevance at the cost of long calculation time [4 [5] 6]. MTM was originally developed to find a golden mean between speed and accuracy [7, later it was extended to non-linear, anisotropic elasticity [8. Recently, linear MTM was successfully applied to cranio-maxillofacial (CMF) surgeries, and validated with 10 clinical cases both in quantitative and qualitative ways 9 . However, most of the previous works have failed to be seamlessly integrated into the clinical workflow in two aspects. First, the simulation accuracy was not enough for surgeons to realize delicate soft-tissue variation around the errorsensitive regions. Second, it requires laborious pre-processing work and long computation time which cannot be acceptable in daily clinical practice.

T. Jiang et al. (Eds.): MICCAI 2010, Part I, LNCS 6361, pp. 61-68, 2010.

(C) Springer-Verlag Berlin Heidelberg 2010 
In this study, we propose a method for accurate and computationally efficient soft-tissue simulation after CMF intervention, while respecting the current clinical workflow. To overcome the limitation in accuracy, we hypothesize that patient-specific muscle characterization is necessary. We propose an efficient way to incorporate patient-specific facial muscles by morphing a template model to patient data. Transversely isotropic MTM was adopted to consider the directional property of facial muscles, and achieve reasonable computation times. Two different template muscle models were evaluated to measure the sensitivity of muscle template on the simulation result. Additionally, modeling sliding contact around teeth and mucosa was introduced to realize the delicate soft-tissue variation on this area. The simulation accuracy was retrospectively validated with a real clinical case.

\section{Methods}

The overview of our simulation pipeline is depicted in Fig 1, Detailed explanations on featured steps will be given in the following sub-sections.

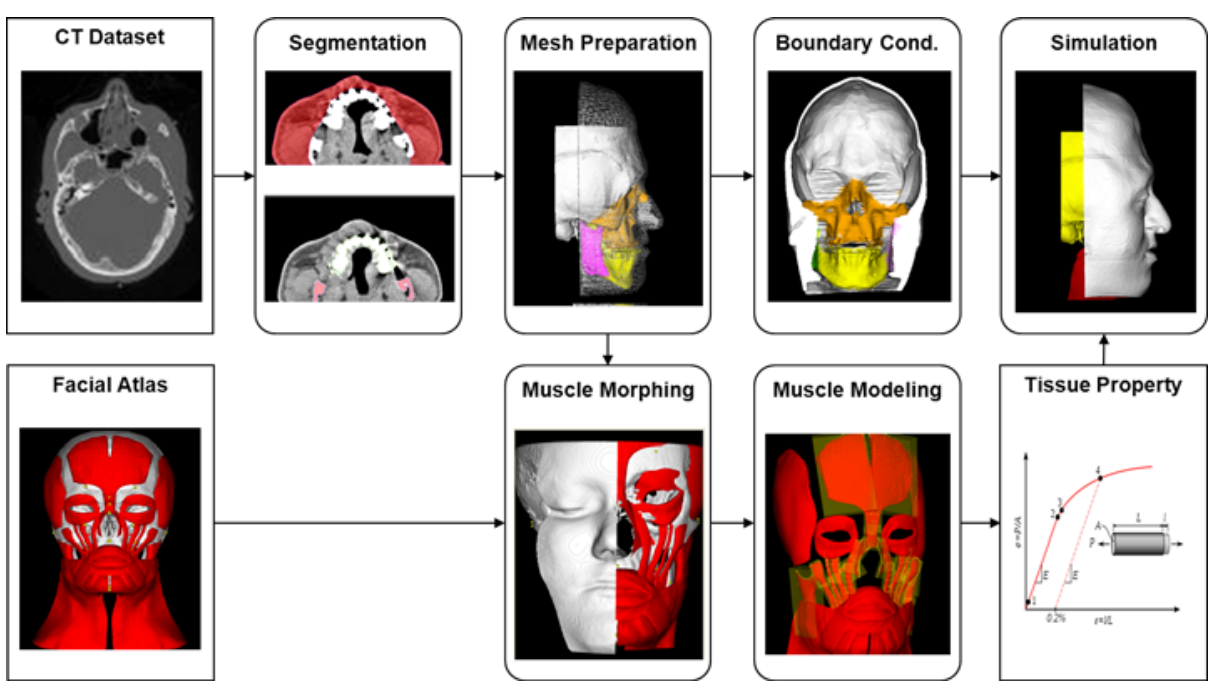

Fig. 1. The pipeline of the anatomically-driven facial soft-tissue simulation method using muscle template model

\subsection{Patient-Specific Muscle Modeling}

Since it is almost impossible to identify individual muscles from clinical Computed Tomographic (CT) scans, we propose to construct patient-specific facial muscles by morphing muscles from facial template models, as shown in Fig.2. 


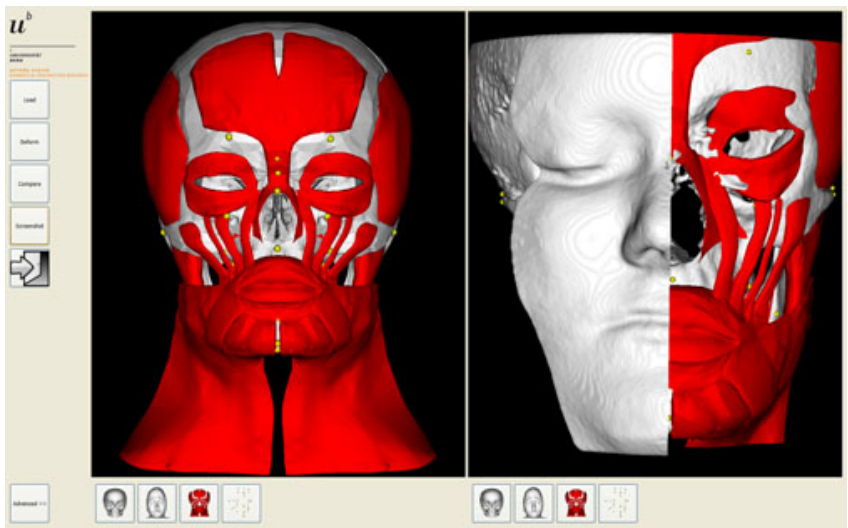

Fig. 2. Morphing of facial muscles from template model (left) to patient-specific anatomy (right), based on skull anatomical landmarks (yellow dots)

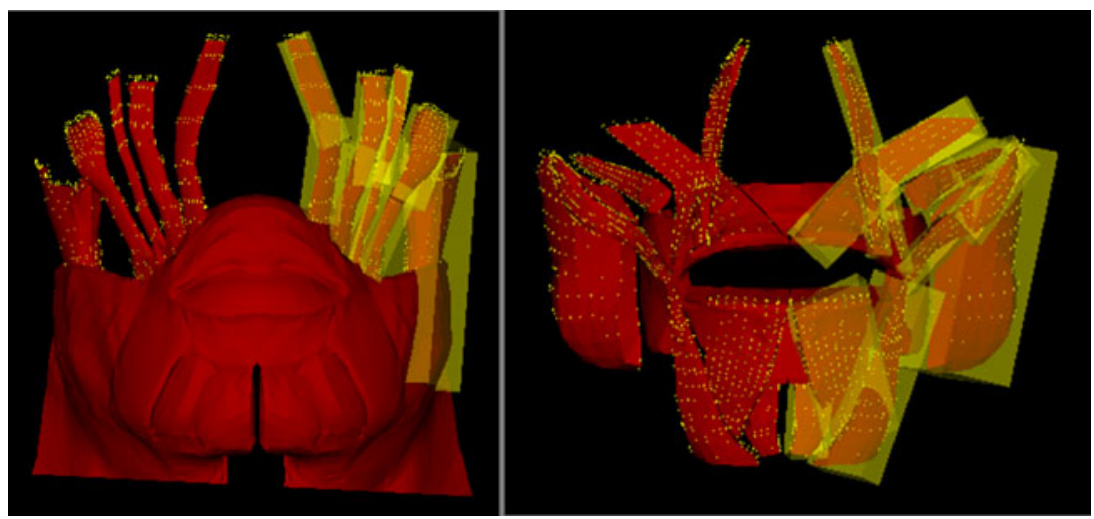

Fig. 3. Extraction of muscle direction using OBB for different muscle templates: muscle template I (left) and muscle template II (right)

For comparison, we evaluated two muscle template models, which consist of different representation of muscles, as shown in Fig 3 . The first template model is a synthetic three-dimensional craniofacial atlas built for educational purposes, and freely available online [10. The second one has more detailed description of individual muscles, since it was obtained by manual segmentation from a high resolution Magnetic Resonance Imaging (MRI) data [11. The morphing procedure was driven by landmark-based thin-plate-spline (TPS) algorithm [12. We adopted thirty-two anatomical landmarks, which are commonly used for measuring skin depth in forensic science [13]. 
In order to obtain the direction of muscles, oriented-bounding boxes (OBB) were extracted for linear-type muscles, then the longitudinal direction of each bounding box was regarded as the direction of muscle for each segment.

\subsection{Material Property Assignment}

To date, there is no consensus in the literature on mechanical properties of facial soft-tissue, since different material parameters can be obtained depending on chosen characterization method and material model. We adopted Young's modulus of muscle tissue along and across fiber from [14. We also refer to the studies of estimating optimal facial tissue properties by comparing the simulated results with real post-operative data, which provides values in ranges [15] [16]. The material properties that we adopted for our simulation are shown in Table 1 . Since there was no information available for directional Poisson ratio of muscle in the literature review, we assumed isotropic Poisson ratio for both directions.

In order to assign appropriate material properties, the intersecting portion of muscle in each tetrahedron needs to be determined. We adopted a random point sampling method inside of each tetrahedron [17, and simple geometrical tests on directional vectors to muscle surface.

\subsection{Boundary Condition Assignment}

We classified vertices on the volumetric mesh into four categories: fixed, joined, sliding and free node, as shown in Fig 4. Fixed nodes are defined on the boundary area, such as the most posterior plane of volumetric soft-tissue. Joined nodes are defined on the interface between movable bony parts and surrounding softtissues. Sliding nodes are specially defined on the area around teeth. Sliding contact has already been tried to simulate the behavior of soft-tissues in breast augmentation [18.

In MTM, the internal force on the $\mathrm{j}$-th node of the volumetric mesh can be given by

$$
\mathbf{f}_{j}=\mathbf{K}_{j j} \mathbf{u}_{j}+\sum_{\forall k \in \Psi_{j}} \mathbf{K}_{j k} \mathbf{u}_{k}
$$

where $\Psi_{j}$ is the set of vertices connected to the $\mathbf{j}$-th node, $\mathbf{u}_{j}$ is the displacement vector on $\mathbf{j}$-th node. $\mathbf{K}_{j j}$ and $\mathbf{K}_{j k}$ are the stiffness tensors for vertex and edge respectively, which are only dependent on material properties and the initial mesh configuration.

Table 1. Material properties used for simulation

\begin{tabular}{ccc}
\hline & Young's modulus (MPa) Poisson Ratio \\
\hline Fat & 0.003 & 0.46 \\
Muscle across fiber & 0.79 & 0.43 \\
Muscle along fiber & 0.5 & 0.43 \\
\hline
\end{tabular}



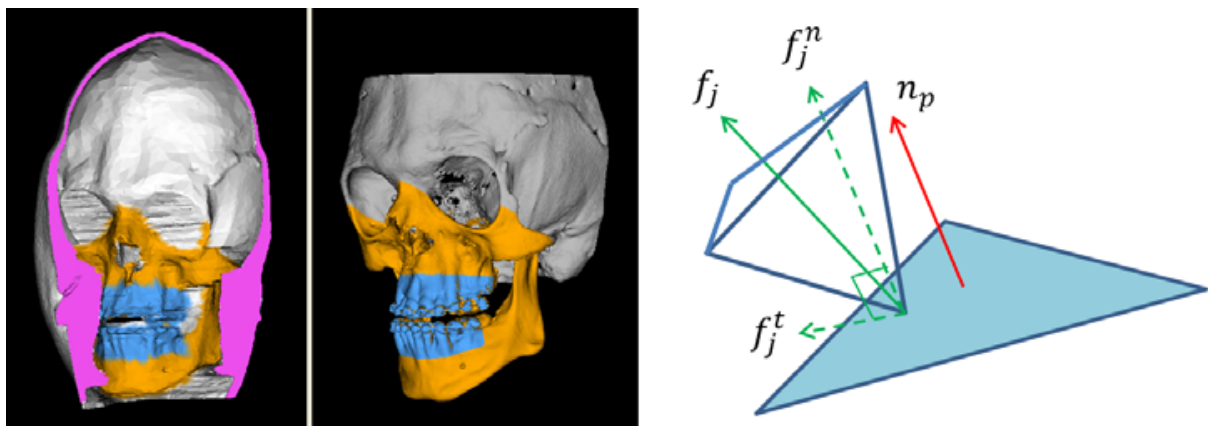

Fig. 4. Classification of volumetric nodes: fixed(pink), joined(orange), sliding(blue) and free(white) (left), force diagram on sliding contact surface (see Eq21) (right).

Especially for sliding nodes, only the tangential component to closest contacting triangle will affect the movement along the contacting surface. No frictional forces are assumed. The tangential force $\mathbf{f}_{j}^{t}$ can be calculated by

$$
\mathbf{f}_{j}^{t}=\mathbf{f}_{j}-\left(\mathbf{f}_{j} \cdot \mathbf{n}_{p}\right) \mathbf{n}_{p}
$$

where $\mathbf{n}_{p}$ is the normal vector of nearest contacting triangle.

\section{Results}

The validation study was retrospectively performed with one clinical case called Pfeiffer-Syndrome. The patient was planned to be treated by Le-Fort III osteotomy with osteodistraction. The amount of maxillary advancement was about $12 \mathrm{~mm}$, while the rotational movement was around 3 degrees. Post-operative CT scan of the patient (in-plane resolution: $0.43 \mathrm{~mm}$, slice distance: $0.6 \mathrm{~mm}$ ) was regarded as a ground-truth to compare simulation accuracy using different tissue models: homogeneous and transversely isotropic elasticity. In addition, we evaluated the simulation results employing two different muscle templates, and sliding contact around teeth and mucosa area. The distance between each simulation and post-operative result was measured based on corresponding points using landmark-based TPS deformation and closest point matching. Fourteen facial anatomical feature points were manually selected for the TPS deformation.

As shown in Fig [5, the median of error was decreased by incorporating transversely isotropy of muscles: homogeneous $(2.36 \mathrm{~mm})$, muscle template I $(2.12 \mathrm{~mm})$. A further improvement was observed using the more detailed muscle template II $(2.01 \mathrm{~mm})$. Higher simulation accuracy was obtained by incorporating sliding contact as additional boundary condition: homogeneous with sliding contact $(1.95 \mathrm{~mm})$, muscle template I with sliding contact $(1.07 \mathrm{~mm})$ and muscle template II with sliding contact $(1.06 \mathrm{~mm})$. The improvement in simulation accuracy was around $1 \mathrm{~mm}$ average, which corresponds to approximately $8 \%$ of 
(a)
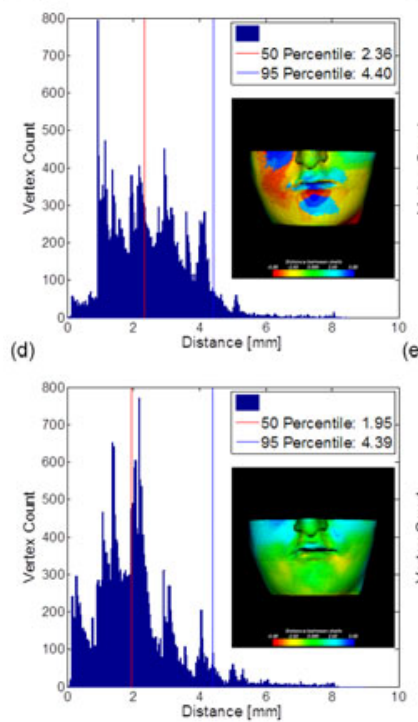

(b)
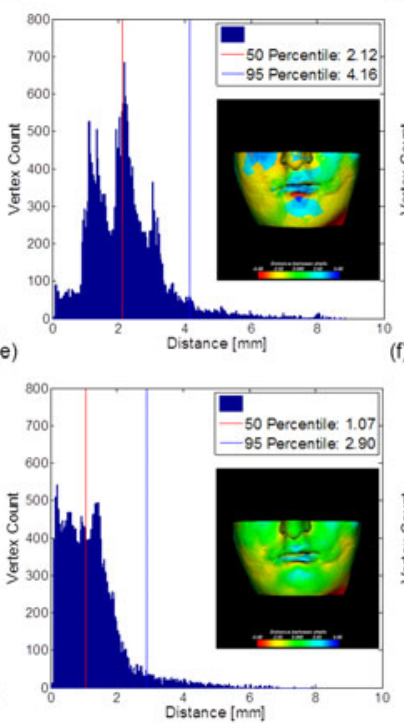

(c)
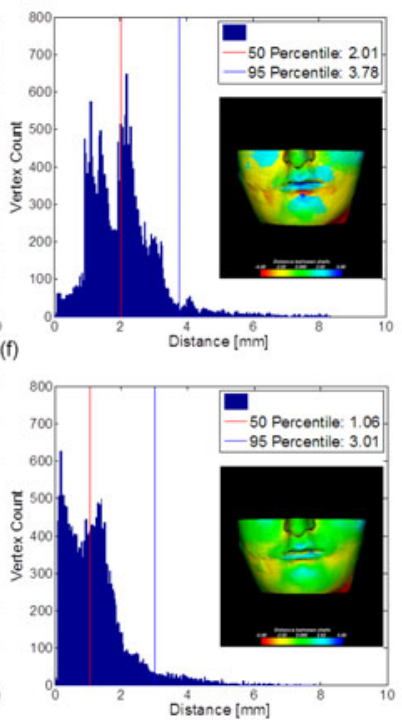

Fig. 5. Comparison of distance errors between simulations and post-operative result: histogram and color maps of (a) homogeneous, (b) muscle template I, (c) muscle template II, (d) homogeneous + sliding contact, (e) muscle template I + sliding contact, (f) muscle template II + sliding contact.

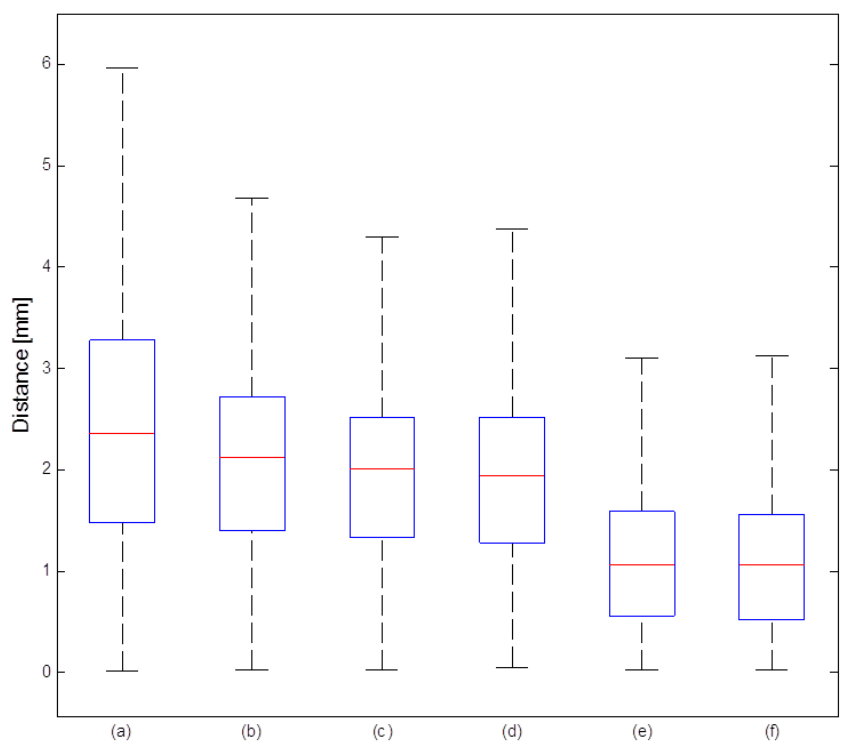

Fig. 6. Box plots of distance errors for the cases presented in Fig [5 box encloses 25th and 75th quartiles, center bar indicates median, whiskers stand for max./min. values excluding outliers 
overall translational movement. This amount of improvements make a difference in the surgeon's perspective, since the improvements occur around nose and lip areas, which are the error-sensitive regions for CMF surgeons. The distribution of the improvements in accuracy can be qualitative assessed by the color-coded distance maps in Fig 5 Statistical relevance was evaluated by Wilcoxon test $(p<0.05)$, which confirmed that there were statistically significant differences in all the pairs of simulated results.

\section{Conclusion}

In this study, we developed an efficient patient-specific soft-tissue simulation method for CMF surgery, with emphasis on its integration into the clinical workflow. A template-based facial muscle morphing technique was proposed to minimize the efforts of segmenting muscle structures without requiring additional imaging modality. A transversely isotropic MTM was applied to realize the directional property of muscle in reasonable calculation time. The retrospective validation study with a real post-operative CT scan confirmed the hypothesis that improvements in accuracy are obtained by introducing template-based facial muscles. Additionally, the results showed that sliding contact was quite effective in increasing simulation accuracy, and so it should be considered in CMF soft-tissue simulations.

As future work, the accuracy of morphing template models to patient-specific anatomy needs to be validated with manual segmentation of the morphology, since it is known that considerable amount of individuality of facial muscle's configuration is present. Additionally, we plan to follow up on the experimental studies on facial tissue characterization to compensate the assumptions of directional properties of muscles in this model. Finally, the extension to non-linear MTM is anticipated to overcome the limited range of deformation provided by linear models, and moreover, the need for non-linear model has yet to be justified for CMF soft-tissue simulations.

\section{Acknowledgments}

This work was funded by the Swiss National Center of Competence in Research "Computer Aided and Image Guided Medical Interventions (Co-Me)", and the AO/ASIF Foundation, Davos, Switzerland.

\section{References}

1. Lee, Y., Terzopoulos, D., Walters, K.: Realistic modeling for facial animation. In: Proceedings of the 22nd annual conference on Computer graphics and interactive techniques, pp. 55-62. ACM, New York (1995)

2. Keeve, E., Girod, S., Kikinis, R., Girod, B.: Deformable modeling of facial tissue for craniofacial surgery simulation. Computer Aided Surgery 3(5) (1998) 
3. Teschner, M., Girod, S., Girod, B.: Direct computation of nonlinear soft-tissue deformation. In: Proc. Vision, Modeling, Visualization, VMV 2000 (2000)

4. Koch, R.M., Gross, M.H., Carls, F.R., von Büren, D.F., Fankhauser, G., Parish, Y.I.H.: Simulating facial surgery using finite element models. In: Proceedings of ACM SIGGRAPH, pp. 421-428. ACM Press, New York (1996)

5. Gladilin, E.: Biomechanical modeling of soft tissue and facial expressions for craniofacial surgery planning. PhD thesis, Free University Berlin (2003)

6. Chabanas, M., Luboz, V., Payan, Y.: Patient specific finite element model of the face soft tissues for computer-assisted maxillofacial surgery. Medical Image Analysis 7(2), 131-151 (2003)

7. Cotin, S., Delingette, H., Ayache, N.: A hybrid elastic model for real-time cutting, deformations, and force feedback for surgery training and simulation. The Visual Computer 16(8), 437-452 (2000)

8. Picinbono, G., Delingette, H., Ayache, N.: Non-linear anisotropic elasticity for realtime surgery simulation. Graphical Models 65(5) (September 2003)

9. Mollemans, W., Schutyser, F., Nadjmi, N., Maes, F., Suetens, P.: Predicting soft tissue deformations for a maxillofacial surgery planning system: from computational strategies to a complete clinical validation. Medical image analysis 11(3), 282-301 (2007)

10. Smith, D.M., Oliker, A., Carter, C.R., Kirov, M., McCarthy, J.G., Cutting, C.B.: A virtual reality atlas of craniofacial anatomy. Plastic and reconstructive surgery 120(6), 1641-1646 (2007)

11. Barbarino, G., Jabareen, M., Trzewik, J., Mazza, E.: Physically Based Finite Element Model of the Face. In: Bello, F., Edwards, E. (eds.) ISBMS 2008. LNCS, vol. 5104, pp. 1-10. Springer, Heidelberg (2008)

12. Bookstein, F.: Principal warps: Thin-plate splines and the decomposition of deformations. IEEE Transactions on pattern analysis and machine intelligence 11(6) (1989)

13. Phillips, V.M., Smuts, N.A.: Facial reconstruction: utilization of computerized tomography to measure facial tissue thickness in a mixed racial population. Forensic Science International 83(1), 51-59 (1996)

14. Duck, F.A.: Physical properties of tissue: a comprehensive reference book. Academic Press, London (1990)

15. Zachow, S., Hierl, T., Erdmann, B.: A quantitative evaluation of 3D soft tissue prediction in maxillofacial surgery planning. In: Proc. of CURAC, pp. 75-79 (2004)

16. Mollemans, W., Schutyser, F., Nadjmi, N., Maes, F., Suetens, P.: Parameter Optimisation of a Linear Tetrahedral Mass Tensor Model for a Maxillofacial Soft Tissue Simulator. In: Harders, M., Székely, G. (eds.) ISBMS 2006. LNCS, vol. 4072, pp. 159-168. Springer, Heidelberg (2006)

17. Uesu, D., Bavoil, L., Fleishman, S., Shepherd, J., Silva, C.: Simplification of unstructured tetrahedral meshes by point sampling. In: Fourth International Workshop on Volume Graphics (2005)

18. Roose, L., De Maerteleire, W., Mollemans, W., Maes, F., Suetens, P.: Simulation of soft-tissue deformations for breast augmentation planning. In: Harders, M., Székely, G. (eds.) ISBMS 2006. LNCS, vol. 4072, pp. 197-205. Springer, Heidelberg (2006) 